\title{
Filigrane
}

Écoutes psychanalytiques

\section{Cet obscur objet de la violence}

\section{Laurence Branchereau}

Volume 25, numéro 1, printemps 2016

Actes du colloque Le sujet sacrifié (Ghyslain Lévy)

URI : https://id.erudit.org/iderudit/1037374ar

DOI : https://doi.org/10.7202/1037374ar

Aller au sommaire du numéro

Éditeur(s)

Revue Santé mentale au Québec

ISSN

1192-1412 (imprimé)

1911-4656 (numérique)

Découvrir la revue

Citer cet article

Branchereau, L. (2016). Cet obscur objet de la violence. Filigrane, 25(1), 67-74.

https://doi.org/10.7202/1037374ar d'utilisation que vous pouvez consulter en ligne.

https://apropos.erudit.org/fr/usagers/politique-dutilisation/ 


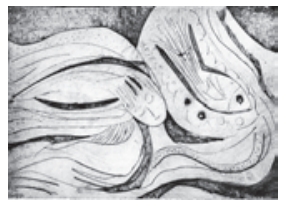

\section{Cet obscur objet de la violence}

\section{Laurence Branchereau}

Outre l'angoisse existentielle de l'homme écrasé dans un milieu déshumanisé par la technologie, nos peurs se nourrissent quotidiennement de la montée de la violence, de l'accroissement des moyens de destruction...

L.V. Thомаs, Traces de mort, mort des traces

Créer, c'est ébranler le sens du monde.

R. BARThes, Sur Racine

Filigrane et L'APPQ nous ont réunis pour discuter autour du thème de la violence qui nous concerne tous, quelles qu'en soient ses racines. Tenter de penser ce qui nous arrive pour essayer «de rendre accessible ce qui des processus psychiques nous semble inaccessible» avec son corollaire, les conduites qui en découlent, est un projet qui sous-tend l'investigation et la méthode psychanalytiques depuis ses débuts.

La violence est un thème actuel tant elle fait irruption dans notre quotidien, celui de la sphère privée, mais aussi via ce que les médias, Internet et l'ensemble des médias sociaux, nous exposent du monde. Les modes d'information sont omniprésents, nous envahissent, mettent à l'épreuve notre capacité de penser. Cet excès, qui insiste, participerait de cette violence par ses effets anesthésiants ou effractants, et pourrait mener à une indifférence, indifférence à l'autre, voire à soi, comme nous le proposent Ghyslain Lévy et Ellen Corin dans des termes différents. Tous deux se sont engagés à cerner les contours de cette violence qui suscite en chacun de nous le trouble et l'effroi.

Violence actuelle, aussi, au sens d'un actuel qui se présente ou se re-présente en séance ou sur la scène externe dans toute sa force, son déséquilibre, ses débordements, ses effets de déliaison, qui déstabilise, désorganise, sidère. Cette violence, par l'inquiétant qu'elle engendre, conduit certains à ne considérer que sa provenance de la scène externe, tentant par là même d'évacuer celle qui se tapit en nous et dont nous ne voulons rien savoir, avec le risque de la répéter inlassablement. Ces restes non traités dont nous parle E. Corin 
en sont une expression, restes qui peuvent s'enkyster ou amener, par un travail d'élaboration, à une remise en mouvement vers la vie.

Une patiente me disait récemment son désarroi en constatant l'indifférence du monde face à la souffrance humaine et à la violence de l'humain faite à l'humain. Elle évoquait le travail obligé des enfants dans certains pays, cette violence exercée sur eux, en lien avec notre consommation des produits bon marché fabriqués dans ces usines qui les enrôlent. "Avec la mondialisation, disait-elle, tout est imbriqué. Nous sommes tous responsables des crimes qui sont commis sur cette planète.» Sentiment de culpabilité qui s'accompagnait d'un autre, et je la cite: «ce trop de responsabilités mène bien des gens à une indifférence et à une insensibilité face à l'autre». Ce questionnement faisait écho à ce qui était sollicité sur la scène intrapsychique et aux traces que ravivaient en elle cette indifférence et cette violence venant du monde.

Ce court fragment de séance reprend, il me semble, ce qu'E. Corin et G. Lévy ont questionné sur le sens et la place à donner à la violence dans la psyché en lien à celle qui nous entoure, à ce qu'elle laisse de traces en nous, et à notre capacité ou non à l'élaborer en fonction de nos expériences, des accidents de l'existence qui ont façonné notre capacité de penser ce qui nous arrive.

Des fils se tendent et s'entrelacent, se déploient entre les propositions de ces deux auteurs D'autres élaborations, en revanche, prennent des couleurs et des textures différentes et, bien qu'elles ne soient pas opposées, semblent à certains égards discordantes. Un certain nombre de questions émerge face à ces contrastes. Néanmoins, $\mathrm{j}$ 'aimerais dans un premier temps énoncer certains points de convergence.

La force du pulsionnel, première violence faite à la psyché, se révèle être un point de jonction, tout comme la place donnée à l'infantile traversé par ce pulsionnel. Cet infantile serait à l'œuvre en sous-main tout au cours de la vie et pourrait se réveiller à tout instant sous l'attraction des chocs provenant tant de l'intérieur que de l'extérieur. En outre, cet infantile met à mal le travail de culture. J’y reviendrai plus loin, car là aussi sont traitées de manière très différente les résonances que la scène externe a sur la psyché individuelle. E. Corin retrace les effets destructeurs de la violence sur la réalité psychique pour dégager notamment, les tentatives d'ouverture face à cette violence qui fait trace, et les possibles reprises de cette violence dans un retour au vivant qui seraient inscrites entre autres dans le travail des artistes. D’un autre côté, G. Lévy met davantage l'accent sur ce qui se referme face à 
cette destructivité, particulièrement dans son texte: Principe d'indifférence et autosacrifice.

La force des pulsions et les traces de cet infantile indomptable auraient partie liées avec la violence. Ce sexuel infantile, qui n'est pas observable, se présente à nous dans ses effets en séance, sur la scène transféro-contretransférentielle, tel que si bien illustré par les auteurs dans leurs fragments de séances, nous rappelant que la règle de libre association favorise la déliaison du pulsionnel, avec ses risques de débordement. À cet égard, E. Corin relève que la langue de l'infantile fait surface à la faveur du transfert, infantile qui déroute certes, mais qui en même temps peut permettre d'animer les traces que la violence a imprimées.

Cette force pulsionnelle donc, dans son débridement, que ce soit sur la scène du monde ou dans la psyché, n'arrive pas toujours à se lier, selon que le sujet soit ou non en état d'impréparation psychique ou qu'il n'ait pas eu, au cours de sa vie, un environnement suffisamment tiercéisant qui lui ait permis de se la représenter. La répétition qui agit compulsivement dans l'après coup viserait la maîtrise de cette excitation.

C'est de manière contrastée que l'un et l'autre des auteurs abordent l'origine pulsionnelle de la violence et de ses effets sur la psyché, ainsi que l'interface entre le dehors et le dedans. Alors que pour E. Corin, c'est à partir du dedans qu'est interrogé, l'impact de ce qui viendrait du dehors, G. Lévy examine essentiellement ce qui du dehors vient imprimer sa marque et s'emparer du dedans, avec les tentatives de déprises qui s'ensuivent. Il invoque toutefois qu'une pulsion d'emprise infantile serait à l'œuvre dans la destructivité, pulsion qui, elle, viendrait du dedans.

Cette pulsion d'emprise, dénuée de compassion à la douleur de l'autre, n'aurait pas pour but la souffrance d'autrui. Cette indifférence libidinale, sans lien avec la haine ou l'amour, serait la marque du narcissisme primaire qui ne prend pas en compte l'objet. Pulsion à visée destructrice de l'objet, d'où proviendrait la pulsion de cruauté infantile qui guide par ailleurs l'élaboration de l'image narcissique en des jeux de retournements et de renversements. G. Lévy propose que, dans un de ces jeux de retournement, une pulsion de contre-emprise tenterait de mettre en échec une auto-emprise sur la vie psychique. Cette dernière se mettrait au service de l'appareil d'emprise, dont il postule l'existence, qui ferait violence à toute vie psychique.

De son côté, E. Corin reprend l'hypothèse d'une pulsion de mort qui s'est imposée à Freud suite aux horreurs vécues durant la Première Guerre mondiale, et qui l'ont mené à un remaniement de sa théorie des pulsions. 
Cette pulsion de mort, en s'intriquant à la pulsion sexuelle, se défléchirait sur le monde ou sur la personne propre. Pulsion qui serait l'expression privilégiée du principe le plus radical du fonctionnement psychique. E. Corin souligne par ailleurs l'importance du masochisme érogène primaire dans sa valence qui le porte à se poser comme gardien de la vie, faisant ainsi barrage à la destructivité issue de la pulsion de mort. Elle ajoute que le masochisme mortifère peut toujours faire surface et provoquer une rupture dans le psychisme pouvant mener à la mort.

Plusieurs questions se posent à partir de ces prémisses. Les premières émanent de l'élaboration de G. Lévy.

En premier lieu, la pulsion d'emprise peut-elle être conçue comme constitutive de la pulsion de mort ou se situe-t-elle sur un autre plan? La pulsion d'emprise évoquée en 1905 dans Les trois essais sur la théorie de la sexualité peut-elle se superposer à celle invoquée dans $A u$-delà $d u$ principe de plaisir? Est-elle de même nature? Cette emprise, co-emprise, autoemprise abordées en retraçant des moments de l'analyse de Vincent, auraitelle comme visée d'empêcher chez l'autre ce qui fait défaut en soi, comme Pontalis l'inférait dans Le mort et le vif entrelacés (1977), ajoutant qu'il y voyait aussi à l'œuvre une emprise du contre-transfert? Cette idée d'emprise du contre-transfert fait-elle écho à ce qui fut éprouvé dans la relation transféro-contretransférentielle avec Vincent? G. Lévy rapporte que Vincent, passivé et contraint dans l'enveloppe de son corps à porter la scène primitive réactualisée dans ce corps à corps avec son frère, passiverait à son tour l'analyste par ce bombardement verbal sexualisé, réactualisant en séance ce trop-plein d'excitations sur la scène séductrice du dispositif analytique qui lui est offert.

Comment faire jouer ces immobilisations provoquées par ces emprises pour remettre en mouvement la vie psychique? Comment résister, dans le but de se dégager de cette visée paralysante, à donner trop rapidement un sens qui fermerait cette éclosion d'un actuel qui déborde en séance?

Par ailleurs, lorsque se présente un effroi partagé en séance face à une violence et un pulsionnel de mort, E. Corin affirme:

[...] c'est par le sensoriel que peut se faire jour quelque chose qui résiste aux représentations et aux mots, un sensoriel qui déroute le champ des représentations initiales et trace la voie au surgissement d'autres images, d'autres mots davantage en prise avec la réalité psychique. De telles images sensorielles sont susceptibles d'animer les tracés figés qu’a imprimés la 
violence dans la psyché, de les détacher de l'effet de sidération accompli par la violence première.

L'auteure insiste sur l'importance de ce sensoriel, cet informe, mais aussi sur la figuration qui peut secondairement se déployer. Elle semble suivre ce fil de la figuration tout le long de son texte, dans un jeu d'ouverture et de fermeture à du nouveau. Quelle est cette part fantasmatique active en chacun de nous, quelles sont ces traces sensorielles, qui font que chaque individu va traiter de manière singulière les évènements de la vie?

La pulsion de mort, bien qu'active en chacun de nous, serait le plus souvent muette, rappelle E. Corin reprenant à son compte l'une des hypothèses formulées par Freud dans Au-delà du principe de plaisir. Concernant cette question du silence de la pulsion de mort, Nathalie Zaltzman (2007), citée par les deux auteurs, quoique dans des registres différents, énonce: «Ce caractère silencieux qu'on lui attribue serait une rétorsion métaphorique [...] de ce qu'elle accomplit: le remplacement par du blanc, une décoloration de l'humeur, par de l'absence, du négatif, du vide, soit toute forme de mise en rupture dans une continuité.»(p. 89). Puis elle ajoute que «[... les figurations de cette activité pulsionnelle abondent à travers la mise en acte de sa décharge, par destruction ou dans la compulsion de répétition» (p. 89). Je retiens de cette longue citation les mots «figurations» et «mise en rupture» qui tendent des fils avec les propositions de G. Lévy et de E. Corin.

André Green postule que la pulsion de mort aurait une fonction désobjectalisante sur l'objet et sur le lien avec l'objet par la déliaison qu'elle provoque. L'objet et les liens à cet objet sont attaqués de ce point de vue. L'objet ne serait donc pas vécu comme indifférent. Ce processus entropique viendrait surtout restreindre le champ de la conflictualisation et la frontière dedans/dehors. Zaltzman, quant à elle, attribuerait dans certains cas à la pulsion de mort une fonction anobjectale: «une volonté de mort à l'état brut, sans autre trait de fonctionnement que la déliaison sous forme de décharge par destruction» (2007, p. 89).

Cette fonction anobjectale serait-elle à mettre en correspondance avec le principe d'indifférence proposé par G. Lévy? Ce principe aurait-il à voir avec une volonté d'anéantissement? L'objet serait-il alors indifférent? G. Lévy souligne que dans l'acte sacrificiel, la part haïe et haineuse de soi, la vie, serait jetée à la face du monde détestée et que ce premier temps de l'autosacrifice serait de se jeter soi-même pour rejeter les autres, dans une confusion entre soi et l'autre. Dominerait alors une forme de principe d'indifférence. 
Cette indifférence pourrait-elle être articulée au moi-réalité initial, comme invoqué par l'auteur, à une période de la vie où le moi se situe dans un état d'indifférenciation avec l'objet? S'agirait-il alors d'une indifférence à l'autre?

G. Lévy écrit par ailleurs, dans Le don de l'ombre, qu'une tyrannie de l'actuel serait une sorte d'emprise vécue par toute une génération d'individus figés dans un actuel qui se joue presqu'exclusivement au niveau du corps, et qui serait sous le signe de la vie et de la mort. Il précise que cette clinique de l'actuel est avant tout celle d'une attaque des liens, surtout des liens de pensée. Faire des liens de pensée, ajoute-t-il, représenterait une menace d'emprise. Se fixer dans un actuel serait l'assurance de n'être soumis à aucun lien. Il poursuit en énonçant que cette volonté de l'individu d'être à soi-même sa propre fin serait l'œuvre vivante de Thanatos. Ces jeunes gens qui se livrent à ces autosacrifices pourraient-ils, sous la force d'attraction de Thanatos, vouloir se dégager de cette emprise par cette mise à mort?

Par ailleurs, comment cette activité pulsionnelle de destruction est-elle repérable dans le cours d'une analyse? La relation d'emprise énoncée par G. Lévy tient-elle lieu de ce repérage? Ou serait-elle plutôt à débusquer du côté du sensoriel comme l'énonce E. Corin en proposant que ce sont les traces de cette violence au plus près du corporel qui nous guident lorsqu'elle survient en séance tant du côté de l'analyste que de celui du patient? Qu'en est-il par ailleurs des effets délétères liés à la fascination et à la jouissance qu'exerce cette violence dans le corps et dans la psyché, dans ce qu'elle imprime de traces à l'insu du sujet, mais aussi du thérapeute; traces et éprouvés corporels qui se réaniment au détour du transfert comme rapporté par les deux auteurs dans leurs cas cliniques? Une autre question se pose alors: de quel type de violence notre écoute peut-elle être aussi porteuse?

Si G. Lévy et E. Corin cherchent à construire des ponts entre la réalité psychique et la culture ou, à tout le moins, à dégager les résonances de l'une sur l'autre, il semble y avoir un décalage, à certains égards, entre les points de vue adoptés pour situer ces effets de résonances. G. Lévy situe son propos du côté des forces destructrices; il dépeint la face sombre d'Internet, ses effets mortifères sur certains individus et la menace que cette Voix d'Internet peut représenter pour le travail de culture. Mais Internet n'ouvrirait-il pas, dans d'autres cas de figure, une brèche face à ces violences destructrices en favorisant une déprise, un dégagement qui trace un sillon d'où peut germer une suture avec le vivant ${ }^{1}$ ?

Certaines images, marquées par la destructivité, renverraient les deux auteurs à une fixation, un arrêt, une fermeture. Ils dénouent des fils qui 
mettent à jour les effets de captation, de fascination et d'attraction liés à la surabondance des images qui matraque, sidère la psyché, de par la cruauté aussi que certaines exhibent. Ces dernières, qui suscitent une attraction malgré l'horreur qu'elles dévoilent, seraient une menace pour le travail de culture. G. Lévy a d'ailleurs livré une remarquable illustration de cette attaque au travail de culture. Du reste, les effets destructeurs sur la vie psychique de cette exposition à l'image, particulièrement lorsqu'elle est frappée du sceau de la solitude et du manque d'accompagnement, de médiation de ces images (comme c'est le cas des adolescents dont parle G. Lévy), sera relevée par les deux auteurs. Ainsi, E. Corin ajoute, en citant Didi Huberman, que la plupart des images qui nous environnent ne proposent qu' «écrans, bouche-trous, sutures par le semblant».

En outre, E. Corin énonce, en revenant sur le destin de l'intrication des pulsions de vie et des pulsions de mort, que si certaines images nous précipitent davantage du côté de Thanatos, certaines œuvres d'artistes permettent le surgissement de traces d'un infigurable de cette violence imprimée au plus près du corps. J'ai été sensible à cette proposition de réouverture à un mouvement de la vie psychique au contact du travail de certains artistes qui nous convoquent par leurs ouvres à animer nos sens et notre pensée à ces tracés gravés par la violence. Le cas du peintre Velickovic, dont j'ai eu l'occasion de voir les peintures, est à cet égard saisissant. La fenêtre entrouverte par ce sensible de la blessure exposée, de la détresse issue de la violence ouvrirait la voie, comme le souligne E. Corin, à un travail de pensée et à une élaboration possible dans un jeu de fermeture et d'ouverture, marque du vivant. Elle fait l'hypothèse que ce travail de remise en mouvement passant par l'informe, le sensoriel et la déroute serait une des voies de reprise du travail de culture. Elle conclut que l'art se fait ainsi l'allié de la psychanalyse sur la scène collective, des alliés dont la culture a bien besoin pour contrer les différents visages que prennent la violence et la destructivité qui en découle. G. Lévy conclut, de son côté, qu'il est essentiel, depuis notre position de psychanalyste, de continuer à nous interroger sur ce conflit entre Éros et Thanatos et sur ses effets sur le travail de culture. G. Lévy et E. Corin ont accompli, tous deux, des pas dans ce sens en nous livrant leurs réflexions.

Laurence Branchereau laurencebranchereau@videotron.ca 


\section{Note}

1. Un peu à la manière de ce que l'auteur rappelle du grand rassemblement de solidarité à la suite des tueries de Charlie Hebdo, facilité par Internet et les médias sociaux, même si certains questionnements peuvent surgir face à ces grandes manifestations qui se tenaient sous la bannière d'un «JE SUIS CHARLIE».

\section{Références}

Freud, S. (1905). Trois essais sur la théorie sexuelle. CEuvres complètes tome VI 1901- 1905. Paris: Presses universitaires de France, 2006.

Freud, S. (1920). Au delà du principe de plaisir. Paris: Presses universitaires de France, 1996. Levy, G. (2015). Le don de l'ombre. Paris: Éditions Campagne Première.

Pontalis, J.-B. (1977). À partir du contre-transfert: le mort et le vif entrelacés (p. 223-240). Entre le rêve et la douleur. Paris: Gallimard.

Zaltzman, N. (2007). Une volonté de mort. Topique, 3 (100), 85-102. 